DOI: $10.3901 / J M E .2019 .11 .169$

\title{
可用于大幅值激励的永磁式非线性隔振器
}

\author{
严 博 ${ }^{1,2}$ 马洪业 ${ }^{1}$ 韩瑞祥 $^{3}$ 王 珂 ${ }^{2}$ 武传宇 ${ }^{1}$ \\ (1. 浙江理工大学机械与自动控制学院 杭州 310018;
}

2. 中国科学院空间应用工程与技术中心太空应用重点实验室 北京 100094;

3. 浙江金火机床有限公司 杭州 311400)

\begin{abstract}
摘要: 大加速度激励可极大影响结构的性能, 甚至造成其破坏, 如火箭发射时产生的强烈振动。提出了一种新型永磁式非线 性隔振器, 利用永磁力构建等效负刚度, 并基于非线性软弹簧实现大加速度激励下的高性能隔振。永磁装置由一个轴向布置 的永磁体和三个沿圆周均布的环形永磁体构成, 所有永磁体均沿轴向磁化。分析并建立了隔振系统理论模型, 基于谐波平衡 法推导并得到了非线性隔振器的传递率表达式。设计了非线性隔振器的试验系统, 分别研究了隔振器在 $0.2 \mathrm{~g} 、 0.3 \mathrm{~g} 、 5 \mathrm{~g}$ 及 $7 \mathrm{~g}$ 加速度激励下的隔振性能。结果表明, 非线性隔振器在大幅值加速度激励 $(7 \mathrm{~g})$ 下具有良好的隔振效果, 其峰值频率降低 $47 \%$, 最大传递率降低 $70 \%$ 。此外, 也研究了永磁装置沿圆周不对称时的隔振特性, 在该情况下, 峰值频率降低 $51 \%$, 最大传递率 降低 86\%。
\end{abstract}

关键词: 非线性隔振; 振动控制; 谐波平衡法; 负刚度; 非线性振动

中图分类号: 0328 ; TH113

\section{Permanent Magnets Based Nonlinear Vibration Isolator Subjected to Large Amplitude Acceleration Excitations}

\section{YAN Bo $^{1,2}$ MA Hongye $^{1}$ HAN Ruixiang ${ }^{3}$ WANG Ke ${ }^{2}$ WU Chuanyu ${ }^{1}$}

(1. Faculty of Mechanical Engineering \& Automation, Zhejiang Sci-Tech University, Hangzhou 310018;

2. Key Laboratory of Space Utilization, Technology and Engineering Center for Space Utilization,

Chinese Academy of Sciences, Beijing 100094;

3. Zhejiang GFIR Machine Tool Co., Hangzhou 311400)

\begin{abstract}
Large amplitude excitations extremely affect the performance of host structures, such as the vibrations during the launch. A novel permanent magnets based nonlinear isolator is proposed, which utilizes the nonlinear force to construct an equivalent negative stiffness and also utilizes the softening spring to realize the high performance isolation. The nonlinear vibration isolator mainly comprises of an axial permanent magnet (PM) and three radial PMs that are uniformly distributed along the circumference, where all the PMs are magnetized along their axes. The governing equation of the isolator is established, and the displacement transmissibility is obtained with the harmonic balance method. The isolation performance tests under the base acceleration with the amplitude of $0.2 \mathrm{~g}$, $0.3 g, 5 g$ and $7 g$ are carried out. The results demonstrate that the peak frequency and corresponding transmissibility decrease by $47 \%$ and $70 \%$ with the nonlinear isolator. For the asymmetric configuration of the radial PMs, the peak frequency and corresponding transmissibility decrease by $51 \%$ and $86 \%$.
\end{abstract}

Key words: nonlinear isolation; vibration control; harmonic balance method; negative stiffness; nonlinear oscillation

\section{0 前言}

有害振动极大影响机器的工作性能, 导致产品 次品率增加，同时，长时间的周期振动将导致疲劳

* 国家自然科学基金资助项目(11602223)。20181229 收到初稿, 20190403 收到修改稿
破坏，对结构产生不可逆的损伤。因此，振动控制 一直是机械工程领域的研究热点。由线性隔振理论 可知, 共振区(即频率小于 $\sqrt{2 \omega_{n}}, \omega_{n}$ 为角固有频率) 的传递比大于 1 , 隔振区 (频率大于 $\sqrt{2 \omega_{n}}$ ) 的传递比 小于 $1^{[1]}$ 。增大隔振器阻尼可降低共振区的响应, 但 会导致隔振区的隔振效果变差 ${ }^{[2-3]}$, 使用线性阻尼并 不能很好的兼顾二者。因此, 国内外学者提出非线 
性阻尼进行隔振控制, 仿真与试验均证明了该手段 可提高全频带的隔振效果 ${ }^{[4-7]}$ 。然而, 非线性阻尼难 于实现, 不易控制及评估, 因此对其应用的要求较 高。线性隔振器可在较低频率下实现隔振, 但是隔 振器刚度降低, 系统的静承载能力下降。

近年来, 利用非线性刚度提高隔振器的隔振能 力的研究得到了广泛关注。VAKAKI 等 ${ }^{[8]}$ 提出了非 线性能量陷概念, 分析了能量跳跃, 并验证了其在 冲击振动下有良好的减振效果。NUCERA 等 ${ }^{[9]}$ 将冲 击振动非线性能量陷应用在两自由度系统并模拟地 震冲击, 利用四次地震数据验证了该方法减振的有 效性。YANG 等 ${ }^{[10-11]}$ 验证了在非线性冲击和不可逆 的目标能量转移情况下, 非线性能量陷可以有效吸 收和耗散航天器的宽频带振动。WANG 等 ${ }^{[12-13]}$ 提出 了一种可应用于建筑物的新型非线性能量陷隔振 器, 并与调谐质量阻尼器进行了对比, 分析了建筑 物在固定和自由平动情况下的减振效果, 证明了该 结构减振效果的优越性。

非线性准零刚度隔振器利用构建等效负刚度特 性, 降低系统固有频率, 提高系统隔振带宽, 从而 提高隔振性能。SUN 等 ${ }^{[14-15]}$ 研究了一类多层剪刀形 桁架机构, 发现其刚度与阻尼均存在非线性, 并可 通过调节机构参数得到优化的非线性参数。LIU 等 ${ }^{[16]}$ 设计了 X 形支撑机构, 并将共振频率降低到 $2 \mathrm{~Hz}$ 以下。ZHOU 等 ${ }^{[17]}$ 利用准零刚度原理研究了凸轮滚轮-弹簧机构, 发现无论激励幅值有多大, 该类减 振器的峰值传递率和起始频率都比线性系统的小。 孙煜等 ${ }^{[18]}$ 利用一对横向放置的线性弹簧与垂向正 刚度线性弹簧并联设计了一种准零刚度隔振器, 可 提高悬挂系统低频阶段的隔振特性及车体运行平稳 性, 改善了乘坐舒适度。刘兴天等 ${ }^{[19]}$ 利用欧拉屈曲 梁结构构建出负刚度, 并与线性隔振器并联使用构 成准零刚度隔振器, 分析了平衡点在刚度零点和偏 离刚度零点下系统的特性。ZHENG 等 ${ }^{[20]}$ 利用两个 同轴且径向磁化的环形永磁体(小永磁体嵌套在大 永磁体内)设计了一种高静刚度低动刚度隔振器, 之 后将其应用在 Stewart 平台上, 验证了该隔振器可有 效降低 Stewart 平台的峰值频率, 并提高其 6 个自由 度的工作性能 ${ }^{[21]}$ 。

以上研究表明, 有效利用非线性特征可提高隔 振元器件的隔振性能。相比利用几何结构产生几何 非线性, 永磁体产生的非线性力具有非接触、响应 快、非线性强、易于集成及成本低等优势。

本文设计了一种永磁式非线性隔振器, 其中, 永磁斥力可实现等效负刚度。相比于利用机械结构 产生负刚度, 本文中的隔振器采用永磁体产生的磁
力获得负刚度降低共振频率, 永磁力的非接触特性 可极大提高隔振器的使用寿命。而且分析现有的文 献可知, 已有的非线性隔振器所取得的良好隔振性 能是基于小加速度激励实现的 ${ }^{[14,16-17,20-22]}$, 例如 $1 \mathrm{~g}$ 以下, 而在大加速度激励情况下系统的响应并未探 讨, 如 $3 g$ 以上。针对该问题, 本文基于新型永磁式 非线性隔振器, 分析了大加速度幅值激励下非线性 隔振器的响应, 试验结果表明在该条件下隔振器依 然能够保证优异的隔振性能。

\section{1 永磁式非线性隔振器结构}

图 1 为永磁式非线性隔振器的 3D 模型和原理 样机, 包括质量-弹簧-阻尼线性单元和非线性单元。 质量-弹簧-阻尼线性单元包括负载面、基平面、三 个相同的线性弹簧和三个用于减小摩擦并提高垂直 方向运动精度的直线轴承, 负载面用于承载负载, 基平面与激振器相连。非线性单元包括一个轴向永 磁体和三组在同一水平面并沿基平面周向均匀分布 的径向永磁体, 轴向永磁体固定在负载面上, 并通

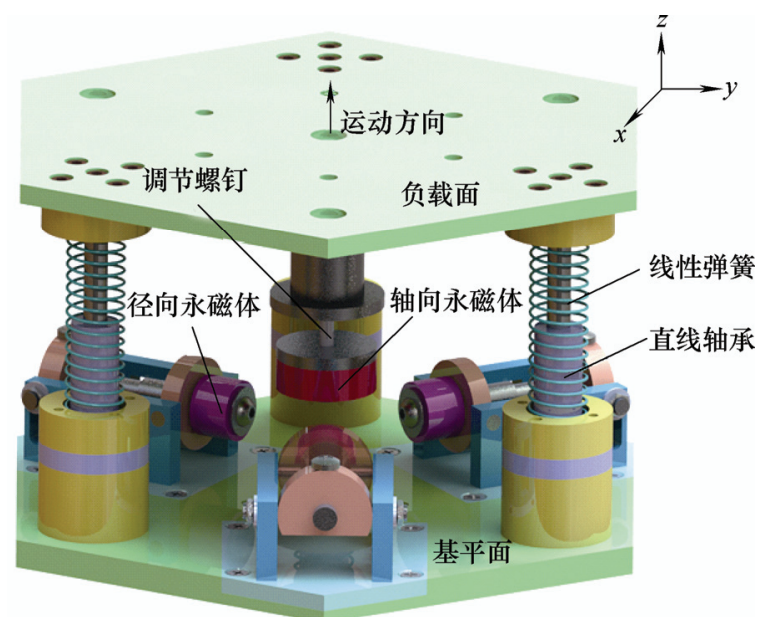

(a) 3D 模型

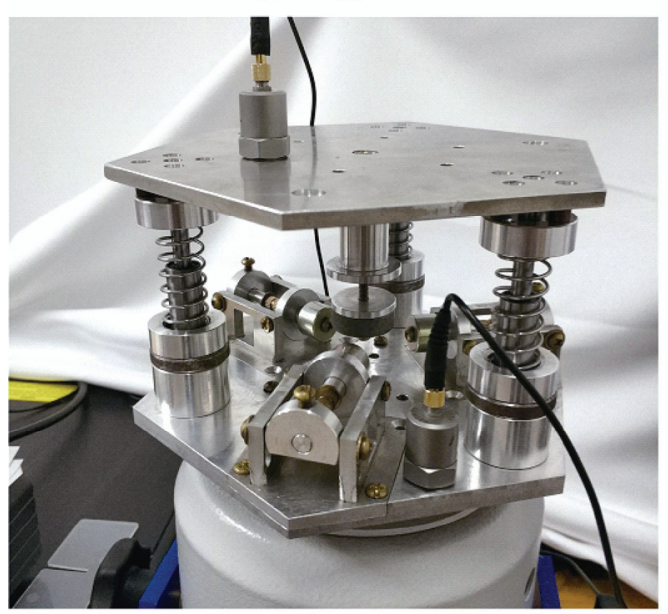

(b) 原理样机

图 1 永磁式非线性隔振器 
过调节螺钉调节其与径向永磁体的垂直高度, 三组 径向永磁体均固定在基平面上。当系统有激励输入 时, 基平面与负载面发生相对运动, 使得轴向永磁 体与径向永磁体发生相对运动。

图 2 为永磁体分布图, 即四个永磁体均沿各自 轴向方向磁化, 轴向永磁体轴线与三个径向永磁体 轴线所在平面垂直, 轴向永磁体与三个均布的径向 永磁体均为斥力。规定: 径向永磁体几何中心到轴 向永磁铁几何中心的垂直距离为 $H$, 水平距离为 $D$ 。 图 1a 中的 “运动方向”与直角坐标系的 $z$ 方向相同。 当轴向永磁体与径向永磁体相斥时, 磁力表现出负 刚度软弹簧的性质, 这减小了系统的刚度, 降低了 峰值频率, 从而提高系统的隔振性能。通过改变 $H$ 和 $D$ 的大小, 可调节负刚度值。
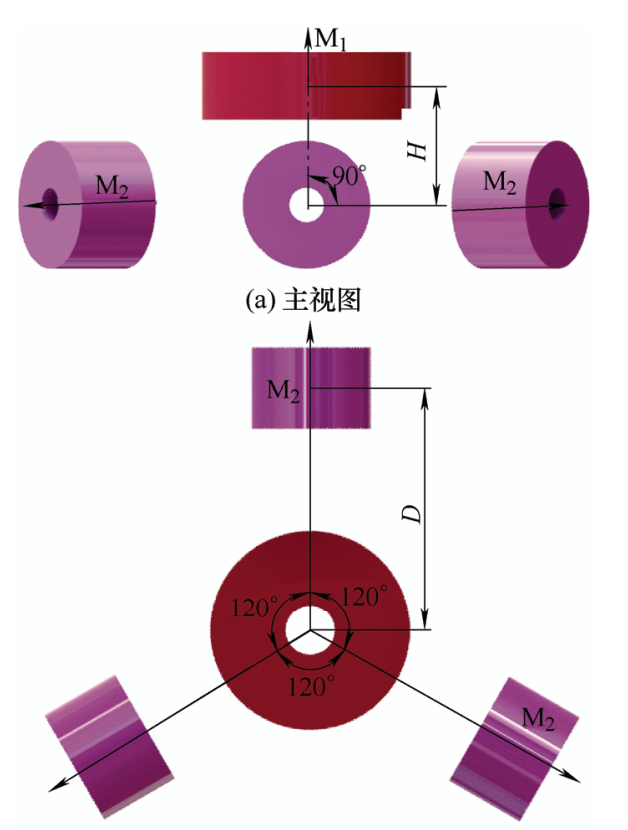

(b) 俯视图

图 2 永磁体分布

\section{2 隔振器理论模型}

\section{1 非线性永磁力分析与简化}

安培电流模型广泛应用于永磁力的计算, 表 1 列出了永磁体的具体参数, 根据文献[22]计算了轴 向永磁体与三个径向永磁体在 $z$ 方向分量的永磁合 力。图 3 给出了在 $D=23.8 \mathrm{~mm}, H=7.5 \mathrm{~mm}$ 的情况 下, 永磁合力随 $z$ 改变而变化的曲线。可知, 在原 点附近, 永磁力的斜率为负, 即可产生等效负刚度。 一般情况下, 磁力随距离的变化可用多项式近似拟 合。而三次方拟合的精度足够, 其表达式如下

$$
F_{m}(z)=f_{c}+k_{1} z+k_{3} z^{3}
$$

式中, $k_{1}$ 和 $k_{3}$ 分别表示永磁合力拟合的线性项系数 和非线性项系数; $f_{c}$ 表示常力, 当系统坐标选取在 静平衡位置时, 可忽略该常力。

表 1 环形永磁体的参数

\begin{tabular}{ccc}
\hline 永磁体参数 & 轴向永磁体 & 径向永磁体 \\
\hline 内径 $/ \mathrm{mm}$ & 2 & 3 \\
外径 $/ \mathrm{mm}$ & 7.50 & 12.25 \\
高度 $/ \mathrm{mm}$ & 10 & 8 \\
剩磁 $/ \mathrm{T}$ & 1.25 & 1.18 \\
\hline
\end{tabular}

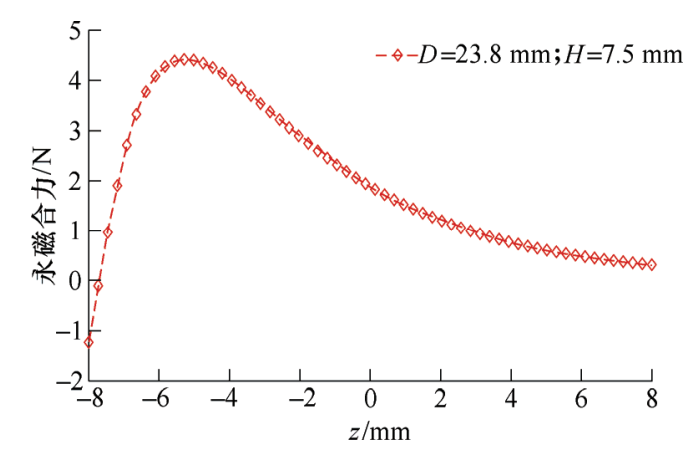

图 3 永磁力仿真曲线

\section{2 单自由度非线性隔振器模型}

图 1 中, 当去除隔振器的三组径向均布的永磁 体结构时, 隔振器为单自由度线性系统。当加入三 组永磁体结构时, 隔振器因引入非线性刚度使得系 统的动力学特性发生改变。图 4 为非线性隔振器简 化模型。右下部分为质量-弹簧-阻尼线性单元, $k$ 表 示三个线性弹簧的总刚度, $C$ 为三个弹簧的等效粘 性阻尼, 根据试验测得的等效粘性阻尼比约为 $0.4 \%$ 。左下部分为永磁结构引入的非线性项。基平 面受到简谐激励作用 $z_{b}(t)$, 经中间隔振单元传递给 质量为 $m$ 的负载面产生相对运动 $z$ 。

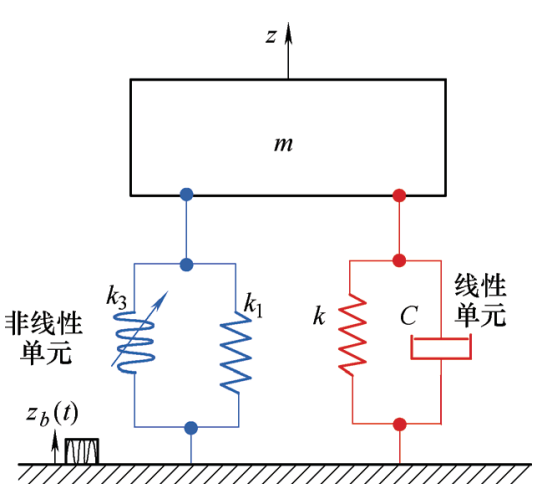

图 4 非线性隔振器的简化模型

非线性永磁力的表达式如式(1), 假设基平面受 到简谐激励为 $z_{b}=Z_{0} \cos (\omega t+\theta)$, 其中, $\omega$ 为外界 激励频率, $Z_{0}$ 为基础激励幅值, $\theta$ 为激励与响应的 相位差。

系统的运动微分方程为 


$$
m \ddot{z}+c \dot{z}+\left(k+k_{1}\right) z+k_{3} z^{3}=-m \ddot{z}_{b}
$$

式中, $m$ 为隔振器的质量, $z$ 为隔振器与基平面的 相对位移。

$$
\begin{aligned}
& \quad \text { 将式(2)量纲一化, 可得 } \\
& \qquad u^{\prime \prime}+2 \xi u^{\prime}+u+\delta u^{3}=\beta^{2} \cos (\beta \tau+\theta) \\
& u=z / Z_{0}, \tau=\omega_{n} t \quad \omega_{n}=\sqrt{\left(k+k_{1}\right) / m} \\
& \xi=c /\left(2 m \omega_{n}\right) \quad \delta=k_{3} Z_{0}^{2} /\left(k+k_{1}\right) \quad \beta=\omega / \omega_{n}
\end{aligned}
$$

式(3)为外界激励为简谐激励的达芬方程, 根据 谐波平衡法, 设方程的解为 $u=U \cos (\beta \tau+\theta)$, 将其 代入式(3)，并忽略高次谐波项，可得

$$
\left\{\begin{array}{l}
-\beta^{2} U+U+\frac{3}{4} \delta U^{3}=\beta^{2} \cos \theta \\
-2 \xi U \beta=-\beta^{2} \sin \theta
\end{array}\right.
$$

隔振器的绝对位移为

$$
\begin{gathered}
z_{2}=z_{b}+z= \\
\left(Z_{0} U+Z_{0} \cos \theta\right) \cos (\beta \tau)-Z_{0} \sin \theta \sin (\beta \tau)
\end{gathered}
$$

系统的位移传递函数为

$$
T=\left|\frac{z_{2}}{z_{b}}\right|=\sqrt{1+U^{2}+2 U \cos \theta}
$$

$\cos \theta$ 可从式(5)推导得出, 将 $\cos \theta$ 代入式(7) 中, 则非线性隔振器的位移传递率为

$$
T=\sqrt{1+U^{2}+\frac{2 U}{\beta^{2}}\left(-\beta^{2} U+U+\frac{3}{4} \delta U^{3}\right)}
$$

若非线性系数 $\delta$ 足够小, 系统可近似为线性系 统, 位移传递率为

$$
T=\sqrt{\frac{\left(k+k_{1}\right)^{2}+\omega^{2} c^{2}}{\left(k+k_{1}-m \omega^{2}\right)^{2}+\omega^{2} c^{2}}}
$$

由式(9)可知, 系统的共振频率由于磁力一次方 系数 $k_{1}$ 的引入而变小。

\section{3 数值仿真}

当 $H$ 改变时, 隔振器的隔振性能也相应发生改 变。表 2 给出了隔振器的物理参数, 系统的等效粘 性阻尼比根据试验数据并利用半功率法求得。当 $D=23.8 \mathrm{~mm}, H=7.5 \mathrm{~mm}$ 时, 利用 Matlab 曲线拟合 功能拟合出磁力曲线拟合系数 $k_{1}=797.3$, $k_{3}=-9.178 \times 10^{6}$ 。可知非线性隔振器可提供负刚度, 系统特性类似于具有软弹簧特征的杜芬方程。幅频 响应的仿真结果基于上述数据得出。仿真中的输入 激励为 $0.2 g$ 。
表 2 隔振器物理参数

\begin{tabular}{lc}
\hline 隔振器参数 & 数值 \\
\hline 质量 $/ \mathrm{kg}$ & 0.39 \\
等效粘性阻尼比 & 0.0429 \\
等效刚度 $/\left(\mathrm{N} \cdot \mathrm{m}^{-1}\right)$ & 2001 \\
\hline
\end{tabular}

图 5 为当 $D=23.8 \mathrm{~mm}, H=7.5 \mathrm{~mm}$ 时的隔振器 传递率曲线。从图中可知, 在引入永磁式负刚度后, 隔振器的峰值频率和对应位移传递率明显降低, 隔 振器的隔振带宽显著提高, 随着频率的增加, 隔振 器的隔振性能越来越好。

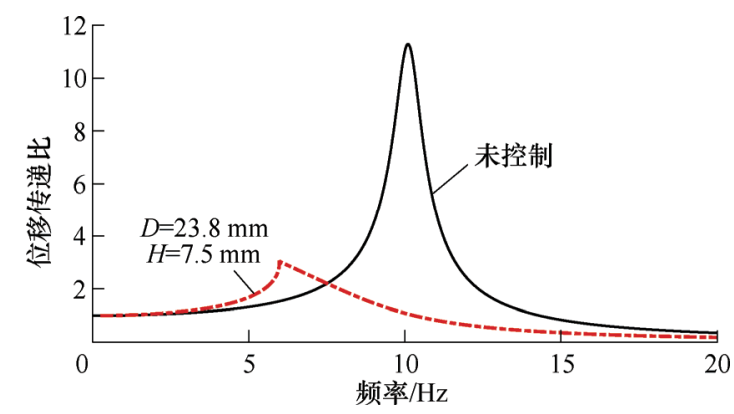

图 5 仿真的传递率曲线

\section{4 隔振器的试验设计与结果分析}

\section{1 试验设计}

图 6 为隔振器测试时的照片。非线性隔振器 的基平面安装在 TIRA 激振器激励平面, 激振器 由控制系统和台面的加速度传感器 1 构成的闭环 系统进行控制。测试所需的激励信号经控制器传 递给功率放大器, 由功率放大器驱动激振器产生 正弦扫频信号。隔振器的响应通过安装在负载面 上的加速度传感器 2 测得, 激振器的输出响应通 过安装在基平面上的加速度传感器 1 测得, 由两 个加速度传感器可得隔振器的传递率。试验中, 正扫频的频率范围为 $[3 \mathrm{~Hz}, 53 \mathrm{~Hz}]$, 扫频时间为 1 min。试验中 “未控制” 的试验曲线是在去除三组 径向永磁体装置时测量所得到的。

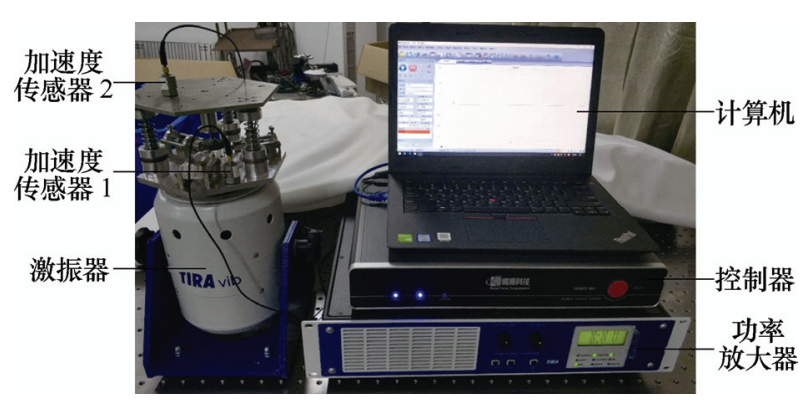

图 6 试验照片 


\section{2 试验结果}

\subsection{1 不同幅值的加速度激励下的隔振性能}

图 7 为 $D=23.8 \mathrm{~mm}, H=7.5 \mathrm{~mm}$ 时, 测试而来 的传递率曲线。此时, 径向永磁体为三个, 外界激 励幅值为 $0.2 \mathrm{~g}$ 。由图可知, 未控制情况下, 隔振器 的峰值频率 $\left(f_{n}\right)$ 和最大位移传递比 $(T)$ 为 $10.72 \mathrm{~Hz}$ 和 11.31 。加入非线性单元后, 峰值频率降低到 $5.30 \mathrm{~Hz}$, 最大位移传递比降低到 2.58 。此时峰值频率降低 $51 \%$, 隔振效果提高 $77 \%$ 。试验结果验证了当径向 永磁体与轴向永磁体之间的磁力为斥力时, 磁力表 现出负刚度软弹簧的性质, 以此降低了系统的峰值 频率与位移传递比, 提高了隔振带宽。

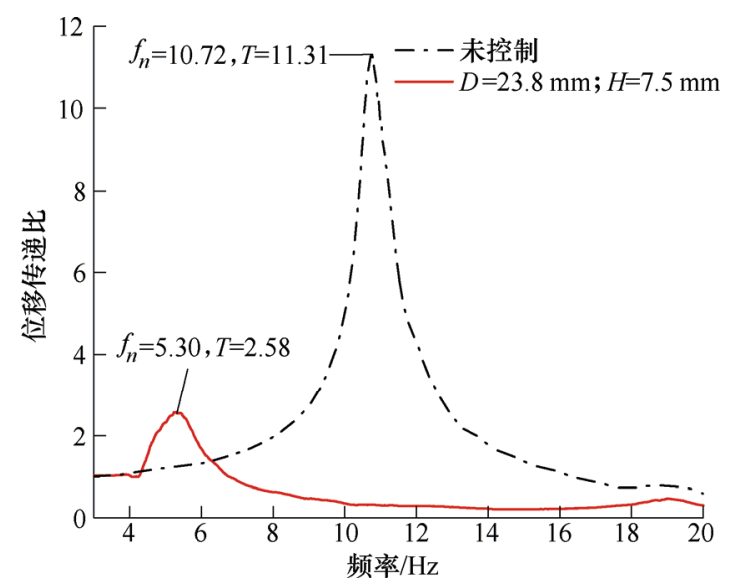

图 7 三组径向永磁体分布的传递率 $(0.2 \mathrm{~g}$ 输入)

图 8 为隔振器在 $5 g$ 和 $7 g$ 激励幅值下的传递 率曲线, 此时 $D=23.8 \mathrm{~mm}, H=7.5 \mathrm{~mm}$, 径向永磁 体为三组。可知, 在 $5 g$ 激励下, 峰值频率降低到 $5.66 \mathrm{~Hz}$ (频率减小 $47 \%$ )、最大位移传递比降低到 3.30 (隔振性能提升 $70 \%$ )。隔振器在 $7 g$ 激励下, 峰值频率降低到 $6.75 \mathrm{~Hz}$ (频率减小 $37 \%$ )、最大位 移传递比降低到 3.47(隔振性能提升 70\%)。试验 结果证明隔振器在大幅值激励下也具有良好的隔 振性能。

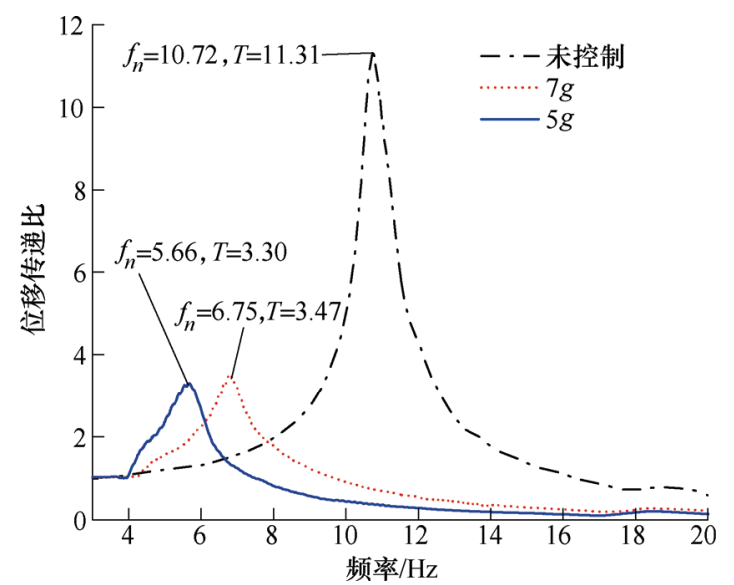

图 8 不同激励幅值下的幅频响应试验结果
4.2.2 磁体沿周向不对称分布时隔振器的隔振性能 当径向永磁体装置由三组变为两组时, 如图 9 所示, 图 10 为测试所得的传递率曲线。此时, $D=22.3$ $\mathrm{mm}, H=6.5 \mathrm{~mm}$, 激励力为 $3 g$ 。由图 10 可知, 相 比于未控制, 隔振器的峰值频率和最大位移传递比 分别降低到 $5.25 \mathrm{~Hz}$ 和 1.55 , 峰值频率降低了 $51 \%$ 、 隔振效果提高了 $86 \%$ 。试验结果验证了当径向永磁 体为两组时, 隔振器隔振性能也有很大提高。但由 于径向永磁体的不对称分布, 对隔振器的安装提出 了更高的要求。

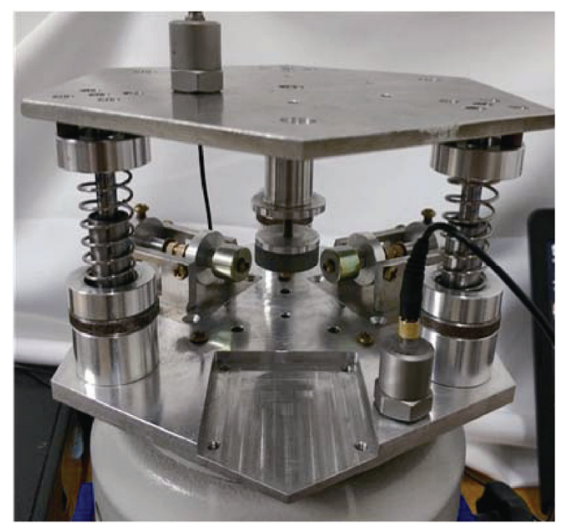

图 9 安装两组径向永磁体图

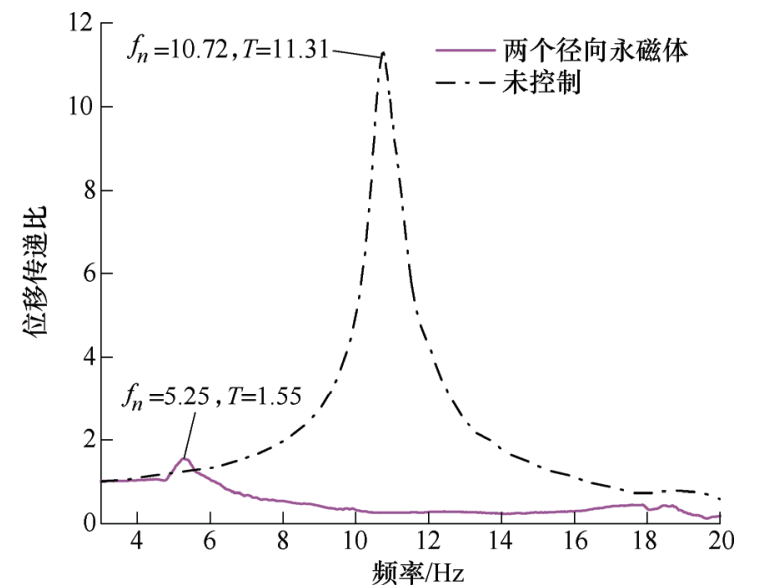

图 10 两组径向永磁体分布的幅频响应试验结果 ( $3 g$ 输入)

\section{5 结论}

(1) 本文提出了一种永磁式非线性隔振器, 由 质量-弹簧-阻尼线性单元和非线性永磁结构单元构 成。基于谐波平衡法获得了隔振器的理论建模及传 递率。仿真结果表明，非线性永磁力可实现等效负 刚度及非线性刚度, 以降低隔振器的峰值频率。

(2) 设计了试验验证系统, 开展了非线性永磁 式隔振器的隔振特性试验研究。结果表明, 安装三 组径向永磁体装置时, 所设计的非线性隔振器可在 小幅值激励和大幅值激励下有效地进行高性能隔 
振，并提高了隔振带宽。

(3) 针对非对称的非线性隔振器, 试验中发现, 其亦可在大幅值激励下进行有效的隔振, 隔振带宽 也同样得到了提高, 表明径向固定的永磁装置的对 称性对隔振器隔振性能影响较小。本文所提出的非 线性永磁式隔振器对航天航空领域及有大幅值激励 的工程应用具有一定的指导意义和参考价值。

\section{参 考 文 献}

[1] PIERSOL A G, HARRIS C M. Harri's shock and vibration handbook[M]. 5th ed. New York: McGraw-Hill, 2017.

[2] YAN B, ZHANG X, NIU H. Vibration isolation of a beam via negative resistance electromagnetic shunt dampers[J]. Journal of Intelligent Material Systems and Structures, 2012, 23(6): 665-673.

[3] YAN B, WANG K, KANG C X, et al. Self-sensing electromagnetic transducer for vibration control of space antenna reflector[J]. IEEE/ASME Transactions on Mechatronics, 2017, 22(5): 1944-1951.

[4] MiLOVANOVIC Z, KOVACIC I, BRENNAN M J. On the displacement transmissibility of a base excited viscously damped nonlinear vibration isolator[J]. Journal of Vibration and Acoustics，2009， 131(5): 054502.

[5] HO C, LANG Z Q, BILLINGS S A. A frequency domain analysis of the effects of nonlinear damping on the duffing equation[J]. Mechanical Systems and Signal Processing, 2014, 45(1): 49-67.

[6] 杜妍辰, 王树林, 朱岩, 等. 带颗粒减振剂碰撞阻尼的 减振特性[J]. 机械工程学报, 2008, 44(7): 186-189. DU Yanchen, WANG Shulin, ZHU Yan, et al. Vibration characteristics of a new fine particle impact damping[J]. Chinese Journal of Mechnical Engineering, 2008，44(7): 186-189.

[7] LIN J L, LIU T H, TSAI K C. Real-valued modal response history analysis for asymmetric-plan buildings with nonlinear viscous dampers[J]. Soil Dynamics and Earthquake Engineering, 2015, 77: 97-110.

[8] VAKAKIS A F. Inducing passive nonlinear energy sinks in vibrating systems[J]. Journal of Vibration and Acoustics, 2001, 123(3): 324-332.

[9] NUCERA F, VAKAKIS A F, MCFARLAND D $\mathrm{M}$, et al. Targeted energy transfers in vibro-impact oscillators for seismic mitigation[J]. Nonlinear Dynamics, 2007, 50(3): 651-677.

[10] YANG K, ZHANG Y W, DING H, et al. Nonlinear energy sink for whole-spacecraft vibration reduction[J]. Journal of Vibration and Acoustics，2017， 139(2): 021011.

[11] 杨凯, 张业伟, 陈立群, 等. 基于非线性消振器的空间
结构被动振动抑制[J]. 动力学与控制学报, 2014, 12(3): 205-209.

YANG Kai, ZHANG Yewei, CHEN Liqun, et al. Space structure vibration control based on passive nonlinear energy sink[J]. Journal of Dynamics and Control, 2014, 12(3): 205-209.

[12] WANG J, WIERSCHEM N E, SPENCER JR B F, et al. Track nonlinear energy sink for rapid response reduction in building structures[J]. Journal of Engineering Mechanics, 2014, 141(1): 04014104.

[13] WANG J, WIERSCHEM N, SPENCER B F, et al. Experimental study of track nonlinear energy sinks for dynamic response reduction[J]. Engineering Structures, 2015, 94: 9-15.

[14] SUN X, JING X, XU J, et al. Vibration isolation via a scissor-like structured platform[J]. Journal of Sound and Vibration, 2014, 333(9): 2404-2420.

[15] SUN X, JING X. A nonlinear vibration isolator achieving high-static-low-dynamic stiffness and tunable anti-resonance frequency band[J]. Mechanical Systems and Signal Processing, 2016, 80: 166-188.

[16] LIU C, JING X, LI F. Vibration isolation using a hybrid lever-type isolation system with an X-shape supporting structure[J]. International Journal of Mechanical Sciences, 2015, 98: 169-177.

[17] ZHOU J, WANG X, XU D, et al. Nonlinear dynamic characteristics of a quasi-zero stiffness vibration isolator with cam-roller-spring mechanisms[J]. Journal of Sound and Vibration，2015，346: 53-69.

[18] 孙显, 宫岛, 周劲松, 等. 低地板有轨电车准零刚度二 系悬挂系统研究 [J]. 机械工程学报, 2017, 53(8): 132-137.

SUN Yu, GONG Dao, ZHOU Jinsong, et al. Study on second quasi-zero-stiffness suspension of low-floor tramcar[J]. Journal of Mechnical Engineering, 2017, 53(8): 132-137.

[19] 刘兴天, 黄修长, 张志谊, 等. 激励幅值及载荷对准零 刚度隔振器特性的影响 [J]. 机械工程学报, 2013, 49(6): 89-94.

LIU Xingtian, HUANG Xiuchang, ZHANG Zhiyi, et al. Influence of exctitation amplitude and load on the characterisitcs fo quasi-zero stiffness isolator[J]. Journal of Mechnical Engineering, 2013， 49(6): 89-94.

[20] ZHENG Y, ZHANG X, LUO Y, et al. Design and experiment of a high-static-low-dynamic stiffness isolator using a negative stiffness magnetic spring[J]. Journal of Sound and Vibration, 2016, 360: 31-52.

[21] ZHENG Y, LI Q, YAN B, et al. A Stewart isolator with high-static-low-dynamic stiffness struts based on negative 
stiffness magnetic springs[J]. Journal of Sound and Vibration, 2018, 422: 390-408.

[22] YAN B, MA H, ZHAO C, et al. A vari-stiffness nonlinear isolator with magnetic effects: Theoretical modeling and experimental verification[J]. International Journal of Mechanical Sciences, 2018, 148: 745-755.

作者简介: 严博, 男, 1986 年出生, 博士, 副教授。主要研究方向为振 动控制、智能材料与结构及非线性振动与控制等。

E-mail: yanbo@zstu.edu.cn
马洪业, 男, 1994 年出生, 硕士研究生。主要研究方向为非线性振动与 控制。

E-mail: hongyem@foxmail.com

韩瑞祥, 男, 1962 年出生, 学士, 高级工程师。主要研究方向为数控机 床以及自动化智能装载技术。

E-mail: hrxzxr163@163.com

王珂, 男, 1982 年出生, 博士, 研究员, 博士研究生导师。主要研究方 向为空间机电系统、动力学与控制及智能结构技术。

E-mail: wangke@csu.ac.cn

武传宇(通信作者), 男, 1976 年出生, 博士, 教授, 博士研究生导师。 主要研究方向为智能装备技术、机器人、机构学以及机械动力学与控制。

E-mail: cywu@zstu.edu.cn 